\title{
Debate Forum: Levocarnitine Therapy Is Rational and Justified in Selected Dialysis Patients
}

\author{
Brian D. Schreiber \\ Division of Nephrology, Department of Medicine, Medical College of Wisconsin, Milwaukee, Wisc., USA
}

\author{
Key Words \\ Levocarnitine - Intradialytic hypotension • \\ Erythropoietin-resistant anemia $\cdot$ C-reactive protein • \\ Fatty acid abnormalities
}

\begin{abstract}
Carnitine is a metabolic cofactor which is essential for normal fatty acid metabolism. Patients with chronic kidney disease on dialysis have been shown both to suffer from disordered fatty acid metabolism and to have a significant deficiency in plasma and tissue carnitine. Aberrant fatty acid metabolism has been associated with a number of cellular abnormalities such as increased mitochondrial permeability (a promoter of apoptosis), insulin resistance, and enhanced generation of free radicals. These cellular abnormalities have, in turn, been correlated with pathological clinical conditions common in dialysis patients including cardiomyopathy with attendant hypotension and resistance to the therapeutic effect of recombinant human erythropoietin (EPO). In 1999, the Food and Drug Administration approved levocarnitine injection for the prevention and treatment of carnitine deficiency in patients on dialysis based on documentation of free plasma carnitine levels in dialysis patients similar to other serious carnitine deficiency states for which treatment was required. Data analysis per-
\end{abstract}

formed by expert panels convened by both the American Association of Kidney Patients and, subsequently, the National Kidney Foundation recommended a trial of levocarnitine therapy for specific subsets of dialysis patients including those with EPO resistance, dialysisrelated hypotension, cardiomyopathy and muscle weakness. In 2003, the Centers for Medicare and Medicaid services convened a Medical Advisory Committee which established reimbursement on a national level for carnitine-deficient dialysis patients who had either dialysisrelated hypotension or EPO resistance. Recently, a correlation between reductions in hospitalization rates of dialysis patients receiving levocarnitine therapy has been demonstrated in a large retrospective study. Despite data-based recommendations and national reimbursement, only a small minority of dialysis patients have been prescribed a therapeutic trial of levocarnitine. Whereas the reasons for the reluctance of nephrologists to prescribe this therapeutic trial are unclear, possible explanations include a lack of appreciation of the pivotal role played by carnitine in cellular metabolism and the strength of evidence for a substantial deficiency of carnitine in dialysis patients, an underestimation of the prognostic import of EPO resistance and dialysis-related hypotension, inadequate dissemination of the clinical trial data supporting the use of levocarnitine in dialysis patients, and the heterogeneous clinical response of di-

\section{KARGER}

Fax +4161306 1234 E-Mail karger@karger.ch www.karger.com
Brian D. Schreiber, MD, Assistant Clinical Professor

Department of Medicine, Division of Nephrology, Medical College of Wisconsin Metabolism Disease Consultants, 1519 Paynes Point Road

Neenah, WI 54956 (USA)

Tel. +1 920886 1804, Fax +1 920886 1817, E-Mail bdschreib@new.rr.com 
alysis patients to levocarnitine therapy. Difficulties in documenting both initial eligibility and evidence of improvement as a result of therapy may also be a contributing factor. This paper discusses the biological role of carnitine and its particular relevance to dialysis patients. Clinical trial data concerning an effect of therapy on EPO resistance and dialysis-related hypotension are summarized along with a discussion of the logic behind the use of levocarnitine in dialysis. Finally, the difficulties posed by a reimbursement policy based on clinical as opposed to laboratory endpoints and a heterogeneous response to therapy are addressed.

Copyright $(2006$ S. Karger AG, Basel

Levocarnitine is a naturally occurring compound primarily acquired from dietary sources, i.e. red meat and dairy products. Twenty-five percent of carnitine stores are endogenously synthesized in the liver and kidney $[1,2]$. Levocarnitine has three main metabolic functions.

First, levocarnitine is essential for the transport of long-chain fatty acids across the mitochondrial membrane into the inner aspect of the mitochondrion where $\beta$-oxidation occurs $[3,4]$. Since approximately $80 \%$ of metabolic energy used by heart and skeletal muscle is derived from long-chain fatty acids, this function is of particular significance in the metabolism of these two organ systems [5]. A second important function that has been emphasized in more recent work is the role of levocarnitine in exporting unmetabolized acyl derivatives from the mitochondrion. This is particularly relevant in pathological states characterized by the incomplete metabolism of fatty acids such as cardiac ischemia, a prominent comorbidity in patients on dialysis. Kobayashi and Fujisawa [6] documented the accumulation of acyl coenzyme A (acylCoA) moieties in the mitochondria of ischemic perfused dog hearts. Addition of levocarnitine to the perfusate reduced the intramitochondrial concentration of acylCoA species in a dose-dependent manner as well as showing an increase in free CoA necessary for the activation of metabolic pathways for both glucose and fatty acid metabolism. This finding was consistent with earlier studies by Suzuki et al. [7] showing a reduction in acylCoA in ischemic dog hearts with a subsequent increase in ATP production when administering levocarnitine.

The effect of a reduction in intramitochondrial acyl moieties and an increase in free CoA is that the ratio of free CoA to acetylCoA is increased. This ability to 'buff- er' the acyl/free CoA ratio is a third significant biochemical effect of levocarnitine administration [8].

The significance of the exportation of unmetabolized acyl groups from the mitochondrion and the buffering of the acyl/free CoA ratio lies in the fact that the intramitochondrial accumulation of excess acylCoA esters and an excess acyl/free CoA ratio has been associated with deleterious metabolic consequences, particularly in relation to the cardiovascular system. The deleterious effects of excess intramitochondrial accumulation of long-chain acylCoA esters (LCACAE) include several aspects.

\section{Inhibition of Adenine Nucleotide Translocase}

Adenine nucleotide translocase (ANT) is an enzyme system which allows energy generated in the mitochondrion to be made available for critical cytoplasmic processes. LCACAE have been shown to inhibit the activity of the ANT complex. Because LCACAE accumulate within the mitochondrion of the cardiomyocyte during cardiac ischemia, they may be responsible for the reduction in ANT activity seen in that condition [9-11].

\section{Promotion of Apoptosis}

Even a small increase in the degree of cardiac myocyte apoptosis has been associated with congestive heart failure, and direct promotion of cardiac apoptosis in animal models has resulted in a deterioration in cardiac function as well as reduced survival [12]. The pathological consequences of apoptosis are especially pronounced in hemodialysis patients. Indeed, in studies on dialysis patients, soluble Fas, a marker of apoptosis, has been shown to correlate with coronary artery disease, peripheral arterial occlusive disease, atherosclerosis, as well as indices of inflammation [132-16]. A number of pathways mediate the acceleration of cellular apoptosis. Prominent among these is an alteration in mitochondrial permeability when mitochondria are exposed to LCACAE [17].

\section{Impaired Insulin Sensitivity as Measured by}

Hyperinsulinemic Euglycemic Clamp or

\section{Intramyocellular Glucose Uptake}

Two possible mechanisms for the promotion of insulin resistance by LCACAE are the prevention of the translocation of the glucose transporter GLUT 4 receptor from the cytoplasm to the cell membrane and the inhibition of glucose oxidation through the suppression of the activity of the pyruvate dehydrogenase complex seen as a consequence of an increased acyl/free CoA ratio $[18,19]$. The interference by LCACAE with glucose metabolism is particularly relevant to dialysis patients in that patients with 
uremia have demonstrated insulin resistance [20, 21]. Indeed, acute administration of levocarnitine has improved insulin sensitivity as measured by an intravenous insulin tolerance test [22].

\section{Enhanced Generation of Free Radicals}

Research performed in the past few years has demonstrated an excess of products indicative of free radical formation in the plasma of dialysis patients. Moreover, an increase in these products has been independently correlated with both total mortality and cardiovascular events in the dialysis population [23]. Excess free radical production in dialysis patients has been attributed to a number of factors including uremia itself, the effects of dialysis, and either evident or occult inflammation. An additional factor that has been shown to promote the generation of lipid peroxidation products are LCACAE, an effect demonstrated by Mak et al. [24] in isolated sarcolemmeal membranes. When dialysis patients are treated with levocarnitine, the concentration of products of free radical generation has been shown to be reduced with a consequent increase in antioxidant capacity [25]. This will be discussed further in the context of erythropoietin (EPO)-resistant anemia.

\section{Fatty Acid Abnormalities in Chronic Kidney Disease}

It is apparent from the above section that disordered fatty acid metabolism is associated with pathological cellular processes of particular relevance to the cardiovascular system. Given the susceptibility of dialysis patients to cardiovascular disease and skeletal muscle dysfunction, disordered fatty acid metabolism in the dialysis population may be expected to have particularly grave consequences. Indeed, disordered fatty acid metabolism and disposition of fatty acids have been shown to be features of chronic kidney disease (CKD). This disorder has a number of manifestations including elevated plasma fatty acid levels, decreased tissue metabolism of fatty acids, and an alteration in the acyl/free carnitine ratio in CKD patients.

Several studies have documented elevated circulating levels of non-esterified fatty acids in the plasma of dialysis patients. Bartel et al. [26] demonstrated an increase in circulating plasma fatty acids during a 4-hour hemodialysis. Similar increases in plasma fatty acid levels were demonstrated by Maeda et al. [27] and Suzuki et al. [7]. Dialysis is not only associated with quantitative changes in fatty acid levels but also with alterations in the distribution of fatty acid moieties.

There is also evidence of defective tissue metabolism of fatty acids in dialysis patients. Sakurabayashi et al. [28] demonstrated abnormal cardiac muscle metabolism of fatty acids manifest by a reduced washout rate of ${ }^{123} \mathrm{I}-(p$ iodophenyl)-3-(R,S)-methylpentadecanoic acid (BMIPP) from the heart. Siami et al. [29] directly measured fatty acid oxidation within the skeletal muscle cells of dialysis patients and found the rate of oxidation to be approximately $50 \%$ of that of healthy controls.

An elevated ratio of acyl/free carnitine in the plasma indicates an abnormality in fatty acid metabolism, since unmetabolized acyl moieties are exported from the cell as acylcarnitines. The ratio in healthy adult omnivores is approximately $0.15-0.25$. A ratio above 0.4 is indicative of a fatty acid metabolic disorder. In several studies of dialysis patients in which the acyl/free ratio has been reported, it has been markedly elevated, ranging from 0.77 to $0.96[28,30-32]$.

Moreover, there is evidence to suggest that these manifestations of disordered fatty acid metabolism are improved by the administration of levocarnitine to dialysis patients. Maeda et al. [27] documented a significant reduction in intradialytic free fatty acid concentrations in the plasma of hemodialysis patients following 12 weeks of administration of oral levocarnitine. A similar decrease in intradialytic and postdialysis plasma fatty acid levels was recorded by Suzuki et al. [7] when levocarnitine was administered to hemodialysis patients with carnitine deficiency. The reduction in plasma free fatty acids was accompanied by a significant reduction in premature ventricular contractions. In regard to the disordered cardiovascular metabolism of fatty acids seen in dialysis patients, Sakurabayashi et al. [28] repeated BMIPP scans in dialysis patients following a course of levocarnitine therapy. He documented a normalization of the BMIPP washout rate, indicating decreased retention of myocardial fatty acids due to improved myocardial fatty acid metabolism. As to the improvement in the consequences of disordered fatty acid metabolism, Vesela et al. [25] demonstrated a significant reduction in the plasma malondialdehyde level with an associated increase in the total antioxidant capacity in a group of hemodialysis patients following a 6month course of levocarnitine therapy. Given the recent emphasis on the importance of endothelial dysfunction in dialysis-related morbidities, it is of note that levocarnitine administration has also been shown to attenuate the endothelial dysfunction induced by free fatty acid elevation in normal subjects [33]. 


\section{Carnitine Deficiency States}

In order to understand dialysis-related carnitine deficiency, two important aspects of carnitine deficiency must be delineated. First, the requirement for carnitine must be considered in light of its adequacy for metabolism of acyl groups whose presence within cells varies in a number of pathophysiologic states. Second, plasma carnitine levels have often been used by default as a means of categorizing patients as either deficient or replete. These values do not always reflect the tissue carnitine concentrations [34].

Carnitine deficiency states can be either primary or secondary. Primary carnitine deficiency is rare and either systemic or affecting only skeletal muscles. Affected cells lack the capacity to transport carnitine from the plasma into the cell. Because this defect involves renal tubular cells, carnitine is not adequately reabsorbed. The result is a deficiency in all carnitine moieties and impaired cellular metabolism of long-chain fatty acids. Clinical presentations of systemic primary carnitine deficiency include progressive cardiomyopathy, skeletal myopathy, and hypoglycemic encephalopathy $[35,36]$.

Secondary carnitine deficiencies include deficiencies secondary to metabolic disorders such as fatty acid oxidation disorders and disorders due to acquired medical conditions and iatrogenic factors. Acquired medical conditions include the Fanconi syndrome which causes renal carnitine wastage. Iatrogenic factors may include carnitine deficiency associated with administration of drugs such as valproic acid or zidovudine, or as a result of losses incurred during hemodialysis [37, 38]. Secondary carnitine deficiency disorders are often characterized not only by decreased levels of free plasma carnitine but also an increased acyl/free carnitine ratio $(>0.4)$ reflective of defective fatty acid metabolism (see above) [34]. They can be associated with symptoms which include encephalopathy, hypotonia, cardiomyopathy, cardiac arrhythmias and a failure to thrive, as well as laboratory abnormalities including elevated liver function tests and hyperammonemia [39].

Dialysis-related carnitine deficiency shares features of both primary and secondary carnitine deficiency. Like primary carnitine deficiency, patients experience inappropriate elimination of free carnitine in the face of reduced plasma levels due to the obligatory loss of carnitine as part of the dialysis process. Plasma free carnitine levels are decreased by approximately $70 \%$ with each dialysis session, reflecting the similarity of molecular size to creatinine whose dialysance is also approximately 0.7 . When adjusted for the low plasma free carnitine level, the absolute loss of carnitine during dialysis exceeds normal urinary losses. Whereas patients with normal kidney function will reabsorb most carnitine once deficient plasma levels are established, dialysis patients continue to lose carnitine because of the passive nature of the dialysis membrane. When subjected to careful study, the clearance of carnitine on dialysis has been shown to exceed normal urinary clearance $[2,40]$.

Like patients with secondary carnitine deficiency, there is evidence that patients with both CKD and on dialysis have a disorder of fatty acid metabolism which results in carnitine insufficiency. Though numerous studies have demonstrated an absolute deficiency of plasma free carnitine as well as an increased acyl/free carnitine ratio in dialysis patients, to date, a recent prospective study by Evans et al. [31] provides the clearest evidence concerning both the extent of carnitine deficiency in dialysis patients as well as the time course for the evolution of carnitine depletion in patients undergoing dialysis. In this study, blood samples were collected from 21 patients at the inception of dialysis and at frequent prescribed intervals during the first 12 months of hemodialysis therapy. A subset of patients had serial muscle biopsies for carnitine levels as well. An additional group of 20 patients on dialysis for at least 12 months was sampled to measure the extent of carnitine deficiency beyond the first year of dialysis. Control carnitine levels from 60 healthy adult patients were obtained for comparison with the dialysis patients. Evans et al. [31] demonstrated that within the first week of dialysis, plasma carnitine levels had significantly decreased. After 12 months of dialysis, the mean level of plasma free carnitine had fallen by $40 \%$, and patients on dialysis for more than 12 months had a further reduction. During the first 12 months of dialysis, total muscle carnitine level had decreased by $20 \%$. Patients on dialysis for more than 12 months had a $43 \%$ reduction in total muscle carnitine compared with their baseline values. Numerous other studies show evidence of carnitine deficiency in patients on dialysis. In these studies, plasma free dialysis levels have ranged from 19.2 to $32.4 \mu \mathrm{mol} / 1$ (normal level 4050) $[30,41,42]$. The combined data from two large clinical trials of levocarnitine in dialysis patients have shown that $95 \%$ of long-term dialysis patients have a plasma free carnitine level below the normal value [43]. The low levels are still the highest levels patients would have in the interdialytic period. By combining measurements before and after dialysis, Evans et al. [44] were able to determine the area under the curve for plasma free carni- 
tine in the interdialytic period as $16.0 \mathrm{mmol} / \mathrm{l}$. This level is in the same range as that reported in primary carnitine deficiency and carnitine deficiency associated with the Fanconi syndrome [45]. On the basis of these significantly low plasma free carnitine levels, the Food and Drug Administration (FDA) approved the use of levocarnitine for dialysis-related carnitine deficiency in December 1999. The fact that the FDA considered the low level of plasma free carnitine a sufficient justification for carnitine replacement independent of the clinical circumstance in the dialysis population is noteworthy in light of subsequent policies and recommendations that have emerged in regard to the use of carnitine in the CKD population. Indeed, the FDA approval memorandum specifically states that the approval 'rests on a biochemical endpoint which is a plausible surrogate. Furthermore, it would be unethical to subject patients to the risks and discomforts of frank deficiency in order to prove a clinical benefit of carnitine supplementation. Suffice it to say that there is ample evidence that carnitine is an essential metabolic intermediate and that carnitine deficiency (whether primary or secondary) can be a serious and lifethreatening condition' [46].

Nonetheless, when the data concerning the clinical effect of carnitine supplementation in dialysis were systematically reviewed by panels of recognized experts convened by both the American Association of Kidney Patients and, more recently, the National Kidney Foundation, the recommendations for use have been remarkably consistent. Both groups recommended a trial of levocarnitine therapy for dialysis patients suffering from cardiomyopathy, significant muscle weakness, EPO-resistant anemia, and dialysis-related symptoms including intradialytic hypotension [47, 48].

As will be outlined below, the Centers for Medicare and Medicaid Services (CMS) issued a national coverage determination for intravenous levocarnitine in dialysis, effective January 2003, that was more restrictive than the expert panel recommendations but included coverage for dialysis-related hypotension and EPO-resistant anemia [49].

Given the significant physiological role of levocarnitine in cellular metabolism, the documented deleterious effects of levocarnitine deficiency in other disease states, the evidence for disordered fatty acid metabolism in patients with advanced CKD as well as a substantial carnitine deficiency, and the demonstrated ability of carnitine administration to improve plasma fatty acid levels and tissue metabolism in dialysis patients, it is unlikely that the failure to prescribe levocarnitine to dialysis patients derives from a denial of the biological plausibility of a therapeutic benefit. What other reasons might there be for denying a dialysis patient with a covered indication a therapeutic trial of levocarnitine? Among possible reasons might be one of the following: (1) the conditions for which levocarnitine therapy has been shown to be beneficial are of insufficient importance to require therapy; (2) the physiological rationale for the use of levocarnitine in specific subsets of patients has not been appreciated; (3) there is insufficient clinical evidence to suggest a possible benefit; (4) the clinical response to levocarnitine is heterogeneous which precludes a certainty of benefit, and (5) it is too difficult or time consuming to identify patients who might be candidates for a therapeutic trial of levocarnitine.

Since the cost to the patient and the dialysis provider is mitigated by a national policy for coverage of levocarnitine in certain subsets of dialysis patients, it would seem most appropriate to address each of these possible reasons stated above for avoiding a therapeutic trial in the two specific conditions associated with carnitine deficiency that are presently covered by the CMS, i.e. EPO-resistant anemia and dialysis-related hypotension [49].

\section{Significance of Reimbursed Indications}

\section{EPO-Resistant Anemia}

Much has been realized about the importance of EPO resistance since the publication of the original Dialysis Outcomes Quality Initiative anemia guideline and its last update in 2001. That update defined EPO resistance as follows: 'an inadequate response to epoeitin therapy is defined as failure to achieve target $\mathrm{Hgb} / \mathrm{Hct}$ in the presence of adequate iron stores at a dose of 450 units $/ \mathrm{kg} /$ week i.v. or 300 units $/ \mathrm{kg} /$ week SC within 4-6 months, or failure to maintain target $\mathrm{Hgb} / \mathrm{Hct}$ subsequently at that dose' [50].

Since by this definition $96 \%$ of dialysis patients were considered EPO sensitive, EPO resistance would not have appeared to be a widespread problem. Moreover, since EPO is generally considered a safe therapy, this small group of patients could be treated with elevated doses of EPO once the underlying causes listed in the guideline on unresponsiveness had been adequately considered and addressed.

However, the landmark paper by Zhang et al. [51] has greatly refined our appreciation of both the prevalence and prognostic importance of EPO resistance, as well as the need to address the resistance itself as a risk factor 
for mortality. In that paper, Zhang et al. [51] conducted a retrospective examination of administrative claims from the United States Renal Data Systems. Using a Cox proportional hazard regression analysis adjusted for baseline variables, the authors reported that for all cohorts of hematocrit (Hct) from $<30$ to $>39 \%$, mortality increased with the dose of EPO required. For patients who were in the Hct target range of $33-36 \%$, those in the highest quartile of EPO requirement had a mortality rate that was nearly double the rate of those whose requirement was in the lowest quartile. Moreover, patients in the highest quartile required more than 22,068 units per week, an amount substantially below the 450 units $/ \mathrm{kg} /$ week stipulated in the Dialysis Outcomes Quality Initiative for a 70-kg patient. Since achieving the target goal using large doses of EPO was associated with a significant mortality risk, the authors concluded that 'in contrast to conventional wisdom, this study suggests that epoeitin dosing requirements could provide important prognostic information beyond that predicted by hematocrit alone'.

Of note, since the CMS national coverage determination only reimburses for levocarnitine if the achieved Hct is $<30 \%$, the policy itself is inappropriately restrictive in light of what is now known about the context and consequences of EPO resistance.

\section{Dialysis-Related Hypotension}

Nephrologists have long been aware of the immediate morbidities resulting from intradialytic hypotension. Dialysis-related hypotension may be associated with serious consequences such as cardiac, cerebral, gastrointestinal and retinal ischemia, as well as troublesome symptoms such as cramping, disorientation, nausea, vomiting, and diaphoresis. Hypotension may compromise the delivery of adequate dialysis with both short- and long-term consequences. In addition, earlier studies by Port et al [52] demonstrated an association between predialysis hypotension and excess mortality, particularly for patients suffering from concomitant coronary disease, congestive heart failure, or a combination of the two conditions. More recently, two studies have demonstrated an association between intradialytic hypotension and decreased survival. Tisler et al. [53] performed a prospective study of a large cohort of dialysis patients in whom intradialytic hypotension was either frequent, occasional, or absent. Patients who endured frequent intradialytic hypotension had significantly reduced survival compared with those who did not experience intradialytic hypotension. Using multivariate analysis, Tisler et al. [53] showed that the excess mortality was only weakly associated with the hypotension per se but more strongly with conditions for which hypotension was a marker, such as hypoalbuminemia and, most strongly, coronary disease. The excess mortality associated with intradialytic hypotension has been confirmed by a second study by Shoji et al. [54], in which hemodialysis hypotension was shown to be an independent risk factor for increased 2-year mortality in a prospective study involving $>1,000$ patients. Of note, the prognostically important hypotension was defined by systolic and diastolic levels alone, without either requiring an associated symptom or any specific intervention for reversal.

\section{Rationale for Use of Levocarnitine in Specific Clinical Conditions}

\section{EPO-Resistant Anemia}

Whereas Zhang et al. [51] have demonstrated that a higher EPO requirement confers an increased mortality risk, additional insight into the rationale that might guide an approach to EPO resistance is provided in the findings of Ifudu et al. [55], who prospectively studied the relationship between various levels of Hct and survival in a group of hemodialysis patients. He reported that whereas a lower Hct was associated with shortened survival, multivariate analysis showed that after accounting for serum albumin, age, and diabetic status, the Hct per se was not a significant predictor of mortality. He suggested that EPO resistance was a marker for an underlying condition characterized by malnutrition and inflammation. Gallucci et al. [56] studied hemodialysis patients with varying EPO requirements. These patients had a similar and adequate iron status without either aluminum toxicity or poorly controlled hyperparathyroidism. The factors distinguishing patients with higher EPO requirements were increased serum lactate dehydrogenase and elevated red cell membrane malondialdehyde, a product of lipid peroxidation indicative of excess reactive oxygen species. Gunnell et al. [57] showed that serum albumin level and age were the best predictors of $\mathrm{EPO} / \mathrm{Hct}$ in hemodialysis patients and that when albumin was excluded from the analysis, the best predictors of the EPO/Hct index were C-reactive protein (CRP) and ferritin in hemodialysis patients and CRP level in patients on peritoneal dialysis. Together, these data indicate an important role for inflammation and downstream events associated with the inflammatory response such as free radical production in determining the response of dialysis patients to recombinant human eryth- 
ropoietin (rHuEPO) therapy. The effect of lipid peroxidation on EPO responsiveness is likely related to the deformation of the red cell membrane and the increase in its osmotic fragility when exposed to oxidative stress. Studies by Vlassoupoulos et al. [58] and Sotirakopoulos et al. [59] have demonstrated that treatment with levocarnitine may ameliorate these abnormalities within the case of the Sotirakopoulos study, a consequent increase in the achieved Hct on a fixed dose of EPO. The improvement in red cell rheology is likely related to the reduction in indices of lipid peroxidation and free radical production demonstrated by Vesela et al. [25], following a 6-month course of intravenous levocarnitine treatment. However, the compelling argument for the use of levocarnitine in EPO-resistant anemia lies in the fact that lipid peroxidation indicative of excess reactive oxygen species has been associated not only with resistance to the effect of rHuEPO but also with such significant endpoints as reduced survival and an increased prevalence of cardiac disease in dialysis patients [22]. A similar association with both reduced EPO responsiveness as well as decreased dialysis patient survival has been shown for an elevated CRP level [60]. Carnitine therapy is relevant in this regard, as was shown in a recent study by Savica et al. [61]. They performed a prospective placebo-controlled study in which approximately 100 patients were treated for 6 months with either placebo or intravenous levocarnitine. The carnitine-treated group had a significant reduction in the plasma CRP level whereas no change was seen in the placebo-treated patients. The reduction was especially pronounced in those patients whose baseline CRP was most elevated.

Because of the implications of a reduction in reactive oxygen species not only for EPO responsiveness but for cardiovascular morbidity and survival, the rationale for the use of levocarnitine in EPO-resistant patients is that there is not only a reduction in EPO resistance, itself an important prognostic factor, but there is also an effect on pathologic indices that have been associated with the more compelling endpoints of mortality and cardiovascular disease.

\section{Dialysis-Related Hypotension}

A somewhat analogous logic may be applied to the use of levocarnitine in dialysis-related hypotension. Though dialysis-related hypotension is frequently multifactorial, there are substantial data to indicate a significant element of cardiac dysfunction in hypotensive-prone dialysis patients. Furakawa et al. [62] showed that the cardiac status of patients with intradialytic hypotension differed from their normotensive counterparts using a combined index which measured systolic and diastolic function. Poldermans et al. [63] showed that patients who were prone to intradialytic hypotension had a reduced cardiac reserve when subjected to dobutamine stress echocardiography. Because of the substantial morbidity and mortality associated with heart disease in dialysis patients when treating a hypotension-prone dialysis patient, optimal therapy should address both the hypotension per se as well as the associated cardiac disorder.

Though there are many therapeutic approaches for intradialytic hypotension, few of these address the cardiac component. Whereas cold dialysate and vasoconstrictor drugs effect a temporary increase in cardiac output, this effect is mediated by an increase in central blood volume secondary to peripheral vasoconstriction rather than an effect on the cardiac muscle per se. Levocarnitine therapy is unique among measures applied to hypotensive dialysis patients in its documented beneficial effect on cardiac parameters, particularly in regard to an improvement in left ventricular ejection fraction (LVEF). These data will be discussed in greater detail in the section on clinical data (see below). Thus, the logic for using levocarnitine lies not only in its demonstrated ability to impact dialysis-related hypotension per se but, perhaps more importantly, in its effect on the associated cardiac dysfunction that is responsible for the excess mortality associated with hypotension on dialysis. In this regard, levocarnitine does not merely address a symptom of cardiac dysfunction (hypotension) but the underlying cardiac compromise as well.

\section{Supporting Clinical Data}

\section{EPO-Resistant Anemia}

Studies of levocarnitine on the achieved hemoglobin $(\mathrm{Hgb})$ or Hct are unusual since the advent of EPO therapy as common practice is to adjust the EPO dose to maintain the Hct within a defined range. Prior to the availability of EPO, Trovato [64] treated hemodialysis patients with levocarnitine or placebo for a 1-year period. Levocarnitine treatment was associated with a significant increase in Hgb and Hct at 6, 9, and 12 months. Sotirakopoulos et al. [59] studied the effect of 3 months of levocarnitine administration on the Hct level as well as red blood cell deformability in a group of hemodialysis patients on a stable dose of EPO. Deformability of red cells in the patients on dialysis was increased indicating greater rigidity of the red blood cells. After 3 months of levo- 
carnitine, erythrocyte rigidity was reduced with a corresponding reduction in erythrocyte deformability. This was accompanied by a significant increase in the measured Hct compared with values obtained prior to levocarnitine therapy. In a recent prospective placebo-controlled trial by Savica et al. [61] involving $>100$ patients, a 6-month course of intravenous levocarnitine $20 \mathrm{mg} / \mathrm{kg}$ was associated with a significant increase $(p<0.0001)$ in the blood Hgb level in contrast to the unchanged level seen in the control group.

Several studies have examined the effect of levocarnitine therapy on the EPO dose required to achieve a certain level of Hgb or Hct. In a placebo-controlled doubleblind study, Labonia [65] treated 13 patients with $1 \mathrm{~g}$ intravenous levocarnitine after each dialysis for 6 months, and 11 patients were treated with placebo. The outcome measure was the dose of $\mathrm{rHuEPO}$ required to maintain the Hct between 28 and 33\%. All patients had normal iron, folate and $\mathrm{B}_{12}$ status. After 6 months, there was no change in the required EPO dose in the placebo group, whereas the carnitine-treated group had a 38\% reduction in EPO dose. Kletzmayr et al. [66] performed a placebocontrolled randomized, double-blind trial on 40 patients who were on a stable dose of rHuEPO, which demonstrated a statistically significant negative correlation between the plasma free carnitine level achieved and both the rHuEPO dose and EPO resistance index. Vesela et al. [25] administered levocarnitine $15 \mathrm{mg} / \mathrm{kg}$ intravenously to hemodialysis patients for 3 months and compared the response of those treated with a group of untreated controls. In the carnitine-treated group, the Hct rose significantly after 3 months of therapy and rose further after 6 months. The corresponding $\mathrm{rHuEPO}$ requirement in the levocarnitine-treated patients was reduced by approximately $40 \%$ after 6 months of treatment. Three months after discontinuation of levocarnitine, the mean Hct had fallen with a corresponding rise in the rHuEPO requirement [25]. Of note, iron status was controlled in these studies either by excluding iron-deficient patients from the study population or having included patients on a standardized iron replacement protocol.

In view of the different study designs in the clinical studies cited above, it is helpful to examine the results of a systematic analysis of studies of the clinical effect of carnitine in dialysis by Hurot et al. [67] which was designed to account for the heterogeneity of effect size in the various individual studies. Using the EPO resistance index as an outcome measure, the authors concluded that in the aggregate, there was a significant reduction in this measure, which indicated a reduction in EPO resis- tance in association with levocarnitine supplementation in dialysis patients. The strength of the carnitine effect was that a patient in the 50th percentile of EPO dose distribution in the placebo group would reduce his/her dose to the 23rd percentile if treated by levocarnitine [67].

\section{Dialysis-Related Hypotension}

In a double-blind placebo-controlled trial of 6 months of levocarnitine therapy, Ahmad et al. [68] documented a significant reduction in the number of patients experiencing one or more episodes of intradialytic hypotension monthly compared with placebo-treated patients.

In a study using a crossover design, Casciani et al. [69] demonstrated that episodes of hypotension were reduced to a statistically significant degree with the use of levocarnitine at both 30 and 60 days. After carnitine discontinuation, the frequency of hypotensive episodes has risen, and the difference compared with treatment became statistically significant 60 days after discontinuation.

Fujita et al. [70] studied a group of long-term hemodialysis patients with hypotension (systolic blood pressure $<115 \mathrm{~mm} \mathrm{Hg}$ ) prior to dialysis. Half the group was treated with levocarnitine for 12 weeks, while the other half served as untreated controls. After 8 weeks of levocarnitine therapy, the mean arterial pressure had increased significantly compared with untreated controls and increased further when measured at 12 weeks.

In regard to the improvement in cardiac status that provides much of the supportive rationale for the use of levocarnitine in dialysis hypotension, a number of studies have measured the effect of levocarnitine administration on LVEF or fractional shortening by echocardiography. Both measures are indicative of left ventricular systolic function and thought to be particularly relevant in dialysis patients as systolic dysfunction manifest by a low LVEF has been shown to be a risk factor for the development of de novo chronic heart failure in dialysis patients.

Van Es et al. [30] studied 16 patients on hemodialysis prior to and following 3 months of levocarnitine therapy. Patients with symptomatic intradialytic hypotension had a reduced mean LVEF and were carnitine deficient. Levocarnitine therapy was associated with a significant increase in LVEF in the group as a whole. The most pronounced improvement occurred in patients with a lower baseline LVEF in which there was an increase in LVEF from a baseline of 0.30 to a post-levocarnitine value of 0.42 . 
Romagnoli et al. [71] studied the effect of levocarnitine on LVEF in a group of dialysis patients who had significant systolic dysfunction with a baseline LVEF $<0.45$. Most patients were already on conventional therapy for chronic heart failure-angiotensin-converting enzyme inhibitor therapy and digitalis glycosides. Eight months of therapy including levocarnitine $1 \mathrm{~g}$ intravenously after each dialysis were associated with a significant improvement in LVEF from a baseline of 0.32 to a final value of 0.42 . Improvement was apparent by 2 months and reached a plateau at 6 months.

Several other studies including those of Trovato et al. [72], Khoss et al. [73], and Matsumoto et al. [74] have demonstrated an increase in LVEF or fractional shortening in association with levocarnitine therapy in dialysis patients [72-74].

Whereas studies including those of Fagher et al. [75] and Sakurabayashi et al. [28] have not confirmed an increase in EF, in these studies, patients were either treated for a short period of time or had a baseline EF within normal limits. Indeed, all five studies in which patients had a reduced EF at baseline and were treated with levocarnitine for at least 3 months have demonstrated an improvement in the LVEF.

Though LVEF is an important clinical measurement, many recent cardiology studies have preferred to examine 'hard' endpoints such as hospitalization, cardiac events, and mortality. In this regard, Kazmi et al. [76] analyzed data from a large cohort of hemodialysis patients to determine the association between carnitine therapy and the rate of hospitalization and number of hospital days. After applying strict inclusion criteria, 2,967 patients who had received levocarnitine were included in the analysis. The issues of regression to the mean and survival bias were addressed by including in the analysis a group of non-carnitine patients matched for age, gender, race, diabetic status, dialysis vintage, total duration of dialysis and hospitalization rate, and presence of cardiovascular disease. Compared with the hospitalization rate in the 3month period prior to the initiation of carnitine, the adjusted relative risk for hospitalization in the carnitine period decreased significantly. When the multivariate analysis was limited to patients with cardiac disease, the rate of hospitalization was decreased by $34 \%$ at $6-9$ months and by $58 \%$ at $15-18$ months compared with the baseline hospitalization rate prior to the initiation of levocarnitine therapy. This reduction in the rate of hospitalization and hospital days was not seen in the matched non-carnitine group [76].

\section{Heterogeneity of Study Results and Challenge of Clinical Documentation Requirement}

An additional reason for the hesitancy of dialysis providers in prescribing a therapeutic trial of levocarnitine is that not all treated patients have a similar clinical response. Indeed, in all clinical trials of levocarnitine in dialysis, there are patients who improve as well as those with a less robust response. This heterogeneity is likely reflective of the difference between evaluating a therapy using a laboratory-defined endpoint such as achieved $\mathrm{Hgb}$ or parathyroid hormone level versus a purely clinical endpoint such as the frequency of hypotension. Indeed, the biochemical endpoint that is the basis for the FDA approval of levocarnitine in dialysis, the correction of carnitine deficiency, is predictably achieved in all clinical trials of carnitine replacement. However, whenever one attempts to demonstrate a clinical endpoint, the underlying heterogeneity of the patient population becomes a factor. The difficulty in demonstrating a clinical benefit proportionate to laboratory improvement is documented by Painter and Moore [77] in their review of exercise and rHuEPO therapy. Painter summarized the effect of raising Hct levels with EPO using the validated surrogate measure of the level of peak oxygen uptake $\left(\mathrm{VO}_{2}\right.$ peak). The mean value for the increase in $\mathrm{VO}_{2}$ peak with treatment of anemia using rHuEPO was only $18 \%$ despite a Hct increase of 58\%. Whereas in normal patients the fractional change in $\mathrm{VO}_{2}$ peak per change in $\mathrm{Hgb}$ is in the range of $0.5-0.9$, in hemodialysis patients, the fractional change with $\mathrm{rHuEPO}$ therapy has ranged from 0.03 to 0.45. Moreover, Painter and Moore [77] document considerable heterogeneity in the degree to which $\mathrm{VO}_{2}$ peak has been shown to improve with EPO. Robertson et al. [78] showed that treatment of anemia with $\mathrm{rHuEPO}$ was associated with a significant increase in $\mathrm{VO}_{2}$ peak from 14.9 to $17.6 \mathrm{ml} / \mathrm{kg}^{-1} / \mathrm{min}^{-1}$. Pitetti et al. [79] were unable to demonstrate this benefit despite an increase in Hct from $26.7 \pm 3$ to $34.3 \pm 2$ with EPO treatment. Similarly, Thompson et al. [80] were unable to demonstrate an improvement in skeletal muscle metabolism in a small group of dialysis patients despite an increase in Hct associated with $\mathrm{rHuEPO}$ administration. Do these heterogeneous and contradictory clinical data indicate that the use of rHuEPO should be curtailed? Of course not. The disparity in these findings merely reflects the heterogeneity inherent in clinical as opposed to laboratory-based studies. As dialysis patients have serious morbidities that are multifactorial, individuals cannot be expected to respond in a uniform manner. Nephrologists might ques- 
tion the ethics of denying a benefit to a subset of patients merely because not all patients were similarly improved. Indeed, the required evaluation of patient response in the CMS NCD and the 2003 NKF practice recommendations specifically addresses the expected heterogeneity of response to levocarnitine.

An additional consequence of applying a clinical standard to the initiation and continuation of a specific therapy is that screening and tracking patients receiving the therapy is more labor intensive. In order to qualify for reimbursement for levocarnitine, the nephrologists must not only demonstrate that a patient is carnitine deficient by a low plasma level of free carnitine but also that he or she has either responded poorly to a standard dose of EPO or has had two episodes within a 30-day period of hypotension that has interfered with the delivery of the intended dialysis. The root cause of this requirement lies not in the nature of levocarnitine therapy itself, but in the disparity between the reimbursement policy of the CMS and the biochemically based indication granted by the FDA. It is indeed more time intensive to perform clinical evaluation as opposed to laboratory analysis. However, the continued excessive morbidity observed among dialysis patients whose 'numbers' are acceptable speaks for the importance of treatment of additional disease-enhancing factors particular to an individual patient. In the case of levocarnitine, the required assessment is facilitated by the cooperation between the physician and nursing and dietary personnel, as well as the creative use of computerized tracking tools.

\section{Summary}

In summary, an appreciation of the metabolic abnormalities seen in dialysis patients, the specific manner in which these abnormalities are addressed by levocarnitine therapy, and both the clinical trial data and underlying rationale for pursuing a therapeutic trial of levocarnitine would recommend such a trial in the subsets of patients for whom the drug is available. The seriousness of the morbidities addressed by levocarnitine justifies both the effort required to screen and re-evaluate patients as well as a reconsideration of the restrictive nature of the present CMS reimbursement policy in light of the recommendations of the FDA and several panels of respected experts.

\section{References}

1 Brass EP: Pharmacokinetic considerations for the therapeutic use of carnitine in hemodialysis patients. Clin Ther 1995;17:176-185.

$>2$ Evans A: Dialysis related carnitine disorder and levocarnitine pharmacology. Am J Kidney Dis 2003;41(suppl 4):S13-S26.

$\checkmark 3$ Fritz IB: The effect of muscle extracts on the oxidation of palmitic acid by liver slices and homogenates. Acta Physiol Scand 1955;34: 367-385.

4 Loster H: The Role of Carnitine in Metabolism in Carnitine and Cardiovascular Disease. Bocholt, Ponte Press Verlags-GmbH, 2003, p 13.

-5 Bohles H, Evangeliou A, Bervoets K, Eckert I, Sewell A: Carnitine esters in metabolic disease. Eur J Pediatr 1994;153(suppl 1):S57-S61.

-6 Kobayashi A, Fujisawa S: Effect of $L$-carnitine on mitochondrial acylCoA esters in the ischemic dog heart. J Mol Cell Cardiol 1994;26: 499-508.

7 Suzuki Y, Narita M, Yamazaki N: Effects of $L$-carnitine on arrhythmias during hemodialysis. Jpn Heart J 1982;23:349-359.

$>8$ Hoppel C: The role of carnitine in normal and altered fatty acid metabolism. Am J Kidney Dis 2003;41(suppl 4):S4-S12.

$\checkmark 9$ Shug AL, Subramanian R: Modulation of adenine nucleotide translocase activity during myocardial ischemia. Z Kardiol 1987;76(suppl 5):26-33.
10 Woldegiorgis G, Yousufzai K, Shrago E: Stud- 17 Furuno T, Kanno T, Arita K, et al: Roles of ies on the interaction of palmitoyl coenzyme A with the adenine nucleotide translocase. J Biol Chem 1982;257:14783-14787.

11 Paulson DJ, Shug AL: Inhibition of the adenine nucleotide translocator by matrix localized palmitoyl-CoA in rat heart mitochondria Biochim Biophys Acta 1984;766:70-76.

12 Wencker D, Chandra M, Nguyen K, et al: A mechanistic role for cardiac myocyte apoptosis in heart failure. J Clin Invest 2003;111:1497_ 1504.

13 Hebert MJ, Masse M, Vigneault N, Sirois I, Troyanov S, Madore F: Soluble Fas is a marker of coronary artery disease in patients with end-stage renal disease. Am J Kidney Dis 2001:38:1271-1276.

14 Masse M, Hebert MJ, Troyanov S, Vigneault N, Sirois I, Madore F: Soluble Fas is a marker of peripheral arterial occlusive disease in haemodialysis patients. Nephrol Dial Transplant 2002:17:485-491.

15 Troyanov S, Hebert MJ, Masse M, Vigneault N, Sirois I, Madore F: Soluble Fas: a novel predictor of atherosclerosis in dialysis patients. Am J Kidney Dis 2003; 41:1043-1051.

$>16$ Dalboni MA, Sardenberg C, Andreoli MC, et al: Soluble Fas: a novel marker of inflammation in uremia. Artif Organs 2003;27:687691. long chain fatty acids and carnitine in mitochondrial membrane permeability transition.

18 Dresner A, Laurent D, Marcucci M, et al: Effects of free fatty acids on glucose transport and IRS-1 associated phosphatidylinositol 3-kinase activity. J Clin Invest 1999; 103:253-259.

19 Moore KH, Dandurand DM, Kiechle FL: Fasting induced alterations in mitochondrial palmitoyl-CoA metabolism may inhibit adipocyte pyruvate dehydrogenase activity. Int $\mathrm{J}$ Biochem 1992:24:809-814.

20 Mak RH: Insulin resistance in uremia: effect of dialysis modality. Pediatr Res 1996;40:304308.

21 Stefanovic V, Nesic V, Stojimirovic B: Treatment of insulin resistance in uremia. Int $\mathrm{J}$ Artif Organs 2003;26:100-104.

>22 Gunal AI, Celiker H, Donder E, Gunal SY: The effect of $L$-carnitine on insulin resistance in hemodialyzed patients with chronic renal failure. J Nephrol 1999; 12:38-40.

23 Scott B, Deman A, Peeters P, et al: Cardiac troponin $\mathrm{T}$ and malondialdehyde modified plasma lipids in haemodialysis patients. Nephrol Dial Transplant 2003;18:737-742.

24 Mak IT, Kramer JH, Weglicki WB: Potentiation of free radical-induced lipid peroxidative injury to sarcolemmal membranes by lipid amphiphiles. J Biol Chem 1986;261:1153-1157. Biochem Pharmacol 2001;62:1037-1046. 
25 Vesela E, Racek J, Trefil L, Jankovy'ch V, Pojer M: Effect of $L$-carnitine supplementation in hemodialysis patients. Nephron 2001;88:218223.

26 Bartel LL, Hussey JL, Shrago E: Effect of dialysis on serum carnitine, free fatty acids, and triglyceride levels in man and the rat. Metabolism 1982;31:944-947.

27 Maeda K, Shinzato T, Kobayakawa H: Effects of $L$-carnitine administration on short-chain fatty acid (acetic acid) and long-chain fatty acid metabolism during hemodialysis. Nephron 1989;51:355-361.

-28 Sakurabayashi T, Takaesu Y, Haginoshita S, et al: Improvement of myocardial fatty acid metabolism through $L$-carnitine administration to chronic hemodialysis patients. Am J Nephrol 1999; 19:480-484.

-29 Siami G, Clinton ME, Mrak R, Griffis J, Stone W: Evaluation of the effect of intravenous $L$ carnitine therapy on function, structure and fatty acid metabolism of skeletal muscle in patients receiving chronic hemodialysis. Nephron 1991;57:306-313.

>30 van Es A, Henny FC, Kooistra MP, Lobatto S, Scholte HR: Amelioration of cardiac function by $L$-carnitine administration in patients on hemodialysis. Contrib Nephrol 1992;98:2835.

-31 Evans AM, Faull RJ, Nation RL, et al: Impact of hemodialysis on endogenous plasma and muscle carnitine levels in patients with endstage renal disease. Kidney Int 2004;6:15271534.

-32 Lennon DL, Shrago E, Madden M, Nagle F, Hanson P, Zimmerman S: Carnitine status, plasma lipid profiles, and exercise capacity of dialysis patients: effects of submaximal exercise program. Metabolism 1986;35:728-735.

-33 Shankar SS, Mirzamohammadi B, Walsh JP Steinberg HO: $L$-carnitine may attenuate free fatty acid-induced endothelial dysfunction. Ann NY Acad Sci 2004; 1033:189-197.

34 Pons R, De Vivo D: Primary and secondary carnitine deficiency syndromes. J Child Neurol 1995;10(suppl 2):S8-S24.

>35 Treem WR, Stanley CA, Finegold DN, Hale DE, Coates PM: Primary carnitine deficiency due to a failure of carnitine transport in kidney, muscle, and fibroblasts. N Engl J Med 1988; 319:1331-1336.

-36 Stanley CA, De Leeuw S, Coates PM, et al: Chronic cardiomyopathy and weakness or acute coma in children with a defect in carnitine uptake. Ann Neurol 1991;30:709-716.

>37 Laub MC, Paetzke-Brunner I, Jaeger G: Serum carnitine during valproic acid therapy. Epilepsia 1986;27:559-562.

-38 Dalakas MC, Leon-Monzon ME, Bernardini I, Gahl WA, Jay CA: Zidovudine-induced mitochondrial myopathy is associated with muscle carnitine deficiency and lipid storage. Ann Neurol 1994;35:482-487.

-39 Hale DE, Bennett MJ: Fatty acid oxidation disorders: a new class of metabolic diseases. J Pediatr 1992;121:1-11.
40 Bohmer T, Bergrem H, Eiklid K: Carnitine deficiency induced during intermittent hemodialysis and renal failure. Lancet 1978;i:126128.

41 Savica V, Bellinghieri G, DiStefano C, et al: Plasma and muscle carnitine levels in haemodialysis with morphological-ultrastructural examination of muscle samples. Nephron 1983; 35:232-236.

42 Wanner C, Forstner-Wanner S, Rossle C, Furst P, Schollmeyer P, Horl WH: Carnitine metabolism in patients with chronic renal failure: effect of $L$-carnitine supplementation. Kidney Int 1987;22(suppl):S132-S135.

43 Fornasini GF, Evans A: Analysis of free carnitine in plasma of ESRD patients undergoing chronic dialysis: what is the best assay? Dial Transplant 2003;32(suppl 1):S2-S1 1.

44 Evans A, Faull R, Fornasini GF, et al: Pharmacokinetics of $L$-carnitine in patients with endstage renal disease undergoing long-term hemodialysis. Clin Pharmacol Ther 2000;68: 238-249.

45 Bernardini I, Rizzo WB, Dalakas M, Bernar J, Gahl WA: Plasma and muscle free carnitine deficiency due to renal Fanconi syndrome. J Clin Invest 1985;75:1124-1130.

46 FDA summary of approval for carnitor injection in dialysis NDA:20-182/s-006, Dec 1999.

47 AAKP Carnitine Renal Dialysis Consensus Group: Role of $L$-carnitine in treating renal dialysis patients. Dial Transplant 1994;23:177181.

48 Eknoyan G, Latos D, Lindberg J, and the National Kidney Foundation Carnitine Consensus Conference: Practice recommendations for the use of $L$-carnitine in dialysis-related carnitine disorder. National Kidney Foundation Carnitine Consensus Conference. Am J Kidney Dis 2003;41:868-876.

49 Medicare Coverage Policy: Levocarnitine for end-stage renal disease decision memorandum, 2002, p 26.

$50 \mathrm{NKF}-\mathrm{K} / \mathrm{DOQI}$ clinical practice guidelines for anemia of chronic kidney disease: update 2000. Am J Kidney Dis 2001;37(suppl 1): S182-S238.

51 Zhang Y, Thamer M, Stefanik D, Kaufman J, Cotter DJ: Epoetin requirements predict mortality in hemodialysis patients. Am J Kidney Dis 2004; 44:866-876.

52 Port FK, Hulbert-Searon TE, Wolfe RA, et al: Predialysis blood pressure and mortality risk in a national sample of maintenance hemodialysis patients. Am J Kidney Dis 1999;33:507-517.

53 Tisler A, Akocsi K. Borbas B, Fazakas L, Ferenczi S, Gorogh S, Kulcsar I, Nagy L, Samik J, Szegedi J, Toth E, Wagner G, Kiss I: The effect of frequent or occasional dialysis-associated hypotension on survival of patients on maintenance haemodialysis. Nephrol Dial Transplant 2003; 18:2601-2605.

54 Shoji T, Tsubakihara Y, Fujii M, Imai E: Hemodialysis-associated hypotension as an independent risk factor for two-year mortality in hemodialysis patients. Kidney Int 2004;66: 1212-1220.
5 Ifudu O, Uribarri J, Rajwani I, Vlacich V, Reydel K, Delosreyes G, Friedman EA: Low hematocrit may connote a malnutrition/inflammation syndrome in hemodialysis patients. Dial Transplant 2002;31:845-848.

\$56 Gallucci MT, Lubrano R, Meloni C, et al: Red blood cell membrane lipid peroxidation and resistance to erythropoietin therapy in hemodialysis patients. Clin Nephrol 1999;52:239245.

57 Gunnell J, Yeun JY, Depner TA, Kaysen GA: Acute-phase response predicts erythropoietin resistance in hemodialysis and peritoneal dialysis patients. Am J Kidney Dis 1999;33:6372.

-58 Vlassopoulos D, Hadjiannakos D, Anogitis AG, et al: Carnitine action on red blood cell osmotic resistance in hemodialysis patients. $\mathbf{J}$ Nephrol 2002; 15:68-73.

-59 Sotirakopoulos N, Athanasiou G, Tsitsios T, Stambolidou M, Missirlis Y, Mavromatidis K: Effect of $L$-carnitine supplementation on red blood cells deformability in hemodialysis patients. Ren Fail 2000;22:73-80.

60 Wanner C, Metzger T: C-reactive protein a marker for all-cause and cardiovascular mortality in haemodialysis patients. Nephrol Dial Transplant 2002;17(suppl 8):29-32.

61 Savica V, Santoro D, Mazzaglia G, Ciolino F, Monardo P, Calvani M, Bellinghieri G, Kopple JD: $L$-carnitine infusions may suppress serum $\mathrm{C}$-reactive protein and improve nutritional status in maintenance hemodialysis patients. $\mathrm{J}$ Ren Nutr 2005; 15:225-230.

62 Furukawa K, Ikeda S, Naito T, et al: Cardiac function in dialysis patients evaluated by Doppler echocardiography and its relation to intradialytic hypotension: a new index combining systolic and diastolic function. Clin Nephrol 2000;53:18-24.

63 Poldermans D, Man in 't Veld AJ, Rambaldi $\mathrm{R}$, et al: Cardiac evaluation in hypotensionprone and hypotension-resistant hemodialysis patients. Kidney Int 1999;56:1905-1911.

64 Trovato GM: Long-term $L$-carnitine treatment of chronic anemia of patients with end-stage renal failure. Curr Ther Res 1982;31:10421049.

65 Labonia WD: $L$-carnitine effects on anemia in hemodialyzed patients treated with erythropoietin. Am J Kidney Dis 1995;26:757-764.

66 Kletzmayr J, Mayer G, Legenstein E, et al: Anemia and carnitine supplementation in hemodialyzed patients. Kidney Int 1999;69(suppl):S93-S106.

67 Hurot JM, Cucherat M, Haugh M, Fouque D: Effects of $L$-carnitine supplementation in maintenance hemodialysis patients: a systematic review. J Am Soc Nephrol 2002;13:708714.

68 Ahmad S, Robertson HT, Golper T, et al: Multicenter trial of $L$-carnitine in maintenance of hemodialysis patients. 2. Clinical and biochemical effects. Kidney Int 1990;38:912918 . 
69 Casciani CU, et al: Beneficial effects of $L$-carnitine in post-dialysis syndrome. Curr Ther Res 1982;32:116-127.

70 Fujita Y, Shinzato T, Takai I, et al: Efficacy of $L$-carnitine administration for long-term dialysis patients with continuous hypotension. Jpn J Artif Organs 1988;17:132-135.

71 Romagnoli GF, Nasco A, Carraro G, Lidestri $\mathrm{V}$ : Beneficial effects of $L$-carnitine in dialysis patients with impaired left ventricular function: an observational study. Curr Med Res Opin 2002; 18:172-175.

-72 Trovato GM, Inetti A, Murgo AM, Carinteri G, Catalano D: Body composition and longterm levo-carnitine supplementation. Clin Ter 1998;149:209-214.

73 Khoss AE, Steger H, Legenstein E, et al: $L$-carnitine therapy and myocardium function in children treated with chronic hemodialysis. Wien Klin Wochenschr 1989;101:17-20.
74 Matsumoto Y, Sato M, Ohashi H, et al: Effects of $L$-carnitine supplementation on cardiac morbidity in hemodialyzed patients. Am J Nephrol 2000;20:201-207.

75 Fagher B, Cederblad G, Monti M, et al: Carnitine and left ventricular function in haemodialysis patients. Scand J Clin Lab Invest 1985; 45:193-198.

76 Kazmi WH, Obrador GT, Sternberg M, Lindberg J, Schreiber B, Lewis V, Pereira BJ: Carnitine therapy is associated with decreased hospital utilization among hemodialysis patients. Am J Nephrol 2005;25:106-115.
77 Painter P, Moore GE: The impact of recombinant human erythropoietin on exercise capacity in hemodialysis patients. Adv Ren Repl Ther 1994;1:55-65.

78 Robertson HT, Haley NR, Guthrie M, Cardenas D, Eschbach JW, Adamson JW: Recombinant erythropoietin improves exercise capacity in anemic hemodialysis patients. Am J Kidney Dis 1990; 15:325-332.

79 Pitetti KH, Ross DL, Campbell KD, Wimberley DK: Exercise capacity of hemodialysis patients following recombinant human erythropoietin treatment. Sports Med Training Rehab 1992;3:261-269.

- 80 Thompson RT, Muirhead N, Marsh GD, Gravelle D, Potwarka JJ, Driedger AA: Effect of anaemia correction on skeletal muscle metabolism in patients with end-stage renal disease: $31 \mathrm{P}$ magnetic resonance spectroscopy assessment. Nephron 1996;73:436-441. 